http://dx.doi.org/10.35381/r.k.v5i3.914

\title{
Business Intelligence aplicado al sector $\underline{\text { Salud }}$
}

\section{Business Intelligence applied to the Health sector}

\author{
Juan Antonio Palacios-Tapia \\ juan.palacios@psg.ucacue.edu.ec \\ Universidad Católica de Cuenca, Cuenca \\ Ecuador \\ https://orcid.org/0000-0003-2266-4804 \\ Juan Carlos Erazo-Álvarez \\ icerazo@ucacue.edu.ec \\ Universidad Católica de Cuenca, Cuenca \\ Ecuador \\ https://orcid.org/0000-0001-6480-2270 \\ Cecilia Ivonne Narváez-Zurita \\ inarvaez@ucacue.edu.ec \\ Universidad Católica de Cuenca, Cuenca \\ Ecuador \\ https://orcid.org/0000-0002-7437-9880 \\ Mireya Magdalena Torres-Palacios \\ mireya.torres@ucacue.edu.ec \\ Universidad Católica de Cuenca, Cuenca \\ Ecuador \\ https://orcid.org/0000-0002-7724-3313
}

Recibido: 20 de mayo de 2020

Revisado: 15 de junio de 2020

Aprobado: 30 de julio de 2020

Publicado: 15 de agosto de 2020 


\title{
RESUMEN
}

Se tuvo por objetivo analizar el business intelligence aplicado al sector de salud basado en una base de datos universal. La investigación fue de tipo descriptiva con diseño de campo no experimental, recopilándose información por medio de 690 funcionarios de la rama médica y odontológica, pública y privada de la ciudad de Cuenca - Ecuador. Un 59.42\% piensa que es muy necesario el archivo de los datos. Normalmente en los centros de salud públicos o privados esta información tiene un almacenamiento físico, por cuanto utilizan formularios de papel para la recopilación. La importancia de crear una solución de inteligencia de negocios, permitiendo que existan alternativas de cómo modernizar la parte administrativa-tecnológica-social, de esta manera generar procesos más rápidos, ágiles, que permitan también respuestas efectivas por parte de los expertos de salud.

Descriptores: Economía de la salud; política de la salud; información económica; toma de decisiones. (Palabras tomadas del Tesauro UNESCO).

\begin{abstract}
The objective was to analyze the business intelligence applied to the health sector based on a universal database. Being of a descriptive type with a non-experimental field design, information was collected through 690 officials of the medical and dental branch, public and private of the city of Cuenca - Ecuador. 59.42\% think that the archiving of the data is very necessary. Normally in public or private health centers this information has a physical storage, since they use paper forms for the compilation. The importance of creating a business intelligence solution, allowing there to be alternatives on how to modernize the administrative-technological-social part, thus generating faster, more agile processes that also allow effective responses from health experts.
\end{abstract}

Descriptors: Health economics; health policy; economic information; decisionmaking. (Words taken from the UNESCO Thesaurus). 


\section{INTRODUCCIÓN}

El sector de la salud tanto pública como privada en el Ecuador desempeña un papel importante en el desarrollo y bienestar de la sociedad a través de los servicios que ellos brindan, sin embargo, estos servicios pueden mejorar. Según informe publicado por el Banco Interamericano de Desarrollo (BID) en el año 2018, colocan al Ecuador por los estudios realizados del año 2017, en el puesto 54 de 71 países analizados, por su ineficiencia en el sector de la salud (BID, 2018). Es por eso que se critica esta ineficiencia indicando que se debería tomar en cuenta la inversión en el sistema de salud no solo a nivel de infraestructura, compras de medicamentos genéricos o sistemas de administración sino en la salud como un proceso preventivo y no correctivo como lo es el enfoque de hoy (Léon, 2018).

Los médicos y odontólogos que laboran a nivel nacional dentro de la red de salud pública como también en el sector privado, forman parte de una población con un número inferior en comparación con la población del Ecuador que tienen que cubrir. El Instituto Nacional de Estadística y Censos INEC, entre los años 2000 y 2017, informa que el número de médicos y odontólogos mantienen una tendencia creciente, con un incremento de 26.989 y 3.514 , respectivamente. La tasa de médicos y odontólogos presentaron una tendencia creciente, especialmente la tasa de médicos, con un incremento de 14 puntos entre el periodo de análisis. Para el año 2017, existen 22 médicos y 3 odontólogos por cada 10.000 habitantes. (Instituto Nacional de Estadística y Censos, 2017). 


\section{Tabla 1}

Número de Médicos y Odontólogos

\begin{tabular}{ccc}
\hline Año & Médicos & Odontólogos \\
\hline 2000 & 10.304 & 1.513 \\
2004 & 12.027 & 1.678 \\
2008 & 15.968 & 2.320 \\
2012 & 24.302 & 3.459 \\
2016 & 33.925 & 4.902 \\
2017 & 37.293 & 5.027 \\
\hline
\end{tabular}

Fuente: Instituto Nacional de Estadística y Censos (2017)

\section{Tabla 2}

Tasa por cada 10.000 habitantes de Médicos y Odontólogos.

\begin{tabular}{ccc}
\hline Año & Médicos & Odontólogos \\
\hline 2000 & 8,22 & 1,21 \\
2004 & 8,87 & 1,24 \\
2008 & 11,03 & 1,60 \\
2012 & 15,66 & 2,23 \\
2016 & 20,52 & 2,97 \\
2017 & 22,23 & 3,00 \\
\hline
\end{tabular}

Fuente: Instituto Nacional de Estadística y Censos (2017).

En el Ecuador se dividen los profesionales del área médica y odontológica por los sectores de salud pública y privada, teniendo en la privada una subdivisión con y sin fines de lucro. En el año 2017, los médicos tienen un 74,37\% dentro del sector público, $19,49 \%$ del sector privado con fines de lucro y un $6,14 \%$ en el sector privado sin fines de lucro. Mientras tanto, los odontólogos se dividen en el $94,12 \%, 3,56 \%$ y $2,32 \%$ 
respectivamente (Instituto Nacional de Estadística y Censos, 2017). Aquí se observa que la concentración de profesionales dentro del área de salud se encuentra en el área pública en ambos casos.

El crecimiento de profesionales dentro del área de salud observada en la información anterior, es también segmentado de manera provincial. La provincia de Morona Santiago, según información publicada por el (INEC, 2017), es la provincia con la cuarta tasa más alta de médicos con un valor del 30.63 por cada 10.000 habitantes. Esta población de profesionales se encuentra dentro del ámbito público y privado con el cual se logra obtener una cobertura en el servicio de salud más extensa e inclusiva por cada habitante de esta provincia.

Aun así, con esta ventaja en cobertura se tiene niveles de ineficiencia inadecuados para un sector tan importante, por cuanto no es lo mismo crecer a nivel de infraestructura de salud cómo tener un nivel alto de eficiencia en la atención brindada en estos centros públicos y privados. Largos turnos, cansancio, estrés, pacientes impacientes, fichas médicas sin información histórica o con información que no se encuentra actualizada de los pacientes, entre otros son considerados como las causas por una atención ineficiente de salud. Esta última causa expresada, es el motivo por el cual se ve la necesidad de desarrollar esta investigación, por cuanto se puede establecer un mecanismo de información universal para el acceso en las diferentes redes de salud, de esta manera el profesional puede tener un panorama amplio del cuadro clínico del paciente para realizar su evaluación, análisis y tratamiento.

En virtud de lo planteado, la investigación tiene por objetivo analizar el business intelligence aplicado al sector de salud basado en una base de datos universal en la ciudad de Cuenca - Ecuador. 


\section{Referencial teórico}

\section{Business intelligence, como una herramienta clave para encaminar una empresa hacia el éxito}

En la actualidad con la tecnología cada día avanzando, las empresas generan una gran cantidad de información o datos de manera diaria, la misma que al saber aprovecharlas, nos permite tener una gran ventaja competitiva que genere estrategias la cual les ayude a crecer. El uso de esta información o datos, para luego ser transformada, filtrada y analizada se convierte en una táctica importante, llamada inteligencia de negocios. Esta herramienta revoluciona a las empresas que tienen dentro de su objetivo crecer y ser más competitivos.

Uno de los primeros en tocar esta herramienta es (Luhn, 1958) que define al sistema de inteligencia de negocio o business intelligence como el poder de aprender de la conexión que se genera con situaciones que encaminen hacia alcanzar un objetivo. En este caso el autor hace referencia a la conexión que se genera con situaciones con la generación que existe de datos o información. En otra referencia se comprende que inteligencia de negocios ayuda a que los responsables de una organización tomen las mejores decisiones por cuanto orientan a la conformación y administración de información mediante el uso de conjuntos de metodologías, prácticas y capacidades (Murillo-Junco \& Cáceres-Castellanos, 2013). Por otra parte, para otro autor business intelligence es el enlace entre hacer una información productiva y las herramientas que se generan para mejorar el servicio de los usuarios (Mora, 2018).

De la misma manera para (Ahumada-Tello \& Perusquia-Velasco, 2016), la inteligencia de negocios es un factor determinante de la competitividad por cuanto la misma se presenta en un adecuado manejo de los sistemas de información, en la innovación y en la gestión de los procesos y de las habilidades que se nacen y se desarrolla dentro de las empresas. El poder de entender la situación actual de la empresa, así como anticiparnos a los acontecimientos futuros permiten tener un conocimiento amplio el momento de una toma de decisión empresarial.

El uso adecuado de estos datos son los que nos facilitan dicha toma de decisión dentro de una empresa (Vanegas-Lago \& Guerra-Cantero, 2013). El business intelligence 
también es definido como una estrategia encaminada a la administración y conocimiento de una empresa, mediante el análisis de los datos generados por la misma (Silva-Idrovo, et al., 2018). En otras palabras, conocer a la empresa y saber que más información necesito de ella, hace que los datos recopilados ayuden a formar estrategias que fortalezcan las decisiones tomadas por los empresarios (Hernández, 2017).

Los gerentes, directores, jefes o dueños de las empresas toman importantes decisiones de manera constante y en muchas de las ocasiones, cuando llegan a su conocimiento algún dilema existente dentro de la misma. En referencia a esto (Rodríguez-Ponce, et al., 2013), manifiestan que como núcleo de la planificación, se encuentra la toma de decisiones, la definen a esta como el proceso que selecciona la dirección de acción a seguir dentro de distintas alternativas posibles, con la necesidad de tomar decisiones acertadas y valiosas para la empresa, también existe la posibilidad de errar con un proceso que genere perdida para la misma. Es por esto la importancia de tener un camino claro a seguir y con el uso de la inteligencia de negocios podemos alcanzar el objetivo deseado.

Comparten una parte importante dentro de las acciones de la inteligencia de negocio los sistemas de información, los procesos de innovación y el desarrollo de procedimientos para obtener un proceso de toma de decisión inteligente. Esta toma de decisión inteligente, dentro de una empresa, son promovidos por los mecanismos, documentos, procesos y políticas (Rocha \& Pereira-Mello, 2016). Una empresa debe estar preparada para asumir o direccionarse hacia el cambio por alguna decisión tomada por sus directivos, sin embargo, todo el equipo debe estar dispuesto y comprometido para ejecutar dicho cambio en las diferentes áreas de la empresa. Esto nos indica que es fundamental manejar un sistema adecuado de comunicación y motivación hacia los trabajadores que harán de la herramienta de inteligencia de negocio establecida, un verdadero éxito.

En diferentes lecturas sobre la inteligencia de negocios se le asocia con la tercera función del ciclo de Deming que es del conocimiento. La inteligencia de negocio tiene como objetivo fundamental la recopilación de datos, extracción de información de 
estos datos y generar conocimiento que es usado en la siguiente función del cuadro de Deming que es de mejora, ayudando a crear proyectos o nuevas estrategias que contribuyen con el crecimiento continuo de la empresa. "No se busca la creación de conocimiento sino se pretende añadir valor" (Nonaka \& Takeuchi, 1995, págs. 59-103). Es precisamente en la gestión del conocimiento donde se sustentan estas estrategias que permiten seguir un conjunto de acciones que la empresa inteligente puede emprender, y que le conceden una ventaja sobre sus competidores, principalmente porque el valor agregado a los servicios o productos que son consecuencia de estas acciones desarrollan una eficiencia en su producción y una eficacia en su funcionamiento que difícilmente pueden ser replicadas por aquellas que no tienen estos procesos ni estrategias definidas (Martínez-Avella \& Wills-Herrera, 2013).

Que no se malinterprete, el business intelligence no está para menospreciar el poder de decisión que tienen los directivos, más bien esta para complementarles, haciendo que las empresas sean más inteligentes, modernas y sobre todo competitivas. Esta herramienta además de ayudar a ahorrar tiempo, lo hace con un mejor y mayor número de información, permitiendo que la toma de decisión sea de un nivel superior. Como menciona (Silva-Solano, 2017) con una mejora en la toma de decisiones en todo nivel, estas herramientas facilitan la selección de datos de manera precisa para poder obtener una conclusión y las acciones que se deben tomar según la actualidad de la empresa. La toma de decisión no solamente es un procedimiento de la alta gerencia, sino además es realizada en diferentes áreas de la empresa por parte de sus responsables. Usando el Business Intelligence no solo facilita obtener opciones para una toma de decisión sino además reduce los margenes de error y riesgos que implica esto por cuanto se basan en análisis estadísticos.

\section{El valor del business intelligence dentro de una empresa}

Hoy en día con un aumento en las fuentes de datos como son el internet, las redes sociales, dispositivos móviles, etc. también se ve aumentado la complejidad de recopilar información importante. Sin embargo, el uso rápido, en línea y en tiempo real, hace que se integren varios procesos para dar real valor y utilidad a esta información. 
La información pasa a ser algo indispensable en la toma de decisiones ya que se convierte en el insumo más importante de una organización (Hugo, et al., 2020). Las herramientas dentro del Business Intelligence han ido evolucionado así como sucede con los datos, ya que son capaces de interpretar y procesar una gran cantidad de información y fuentes para con estos resultados extraer la mejor solución o estrategia para una empresa. Las soluciones generadas mediante la inteligencia de negocios están diseñadas para que los usuarios tengan disponible un análisis con mayor profundidad, una variedad de opciones de almacenamiento y una clara administración cualitativa y cuantitativa de los datos (Fuentes-Tapia \& Valdivia-Pinto, 2010).

Otro punto es el control de costos que ellos explican como el punto que exige a las empresas a implementar una solución de este tipo para poder medir los gastos y poder ver con detalle las líneas del negocio, los productos, los costos implicados, entre varios otros aspectos más. Como tercer beneficio, ellos hacen referencia a que la solución de inteligencia de negocios permite entender mejor a los clientes, por cuanto las empresas guardan una gran cantidad de información valiosa de ellos. Es aquí donde entramos en el proceso de transformar esta información en conocimiento y ese conocimiento en una estrategia que permita tomar una decisión competitiva e importante para la empresa y su crecimiento.

Por último, y como beneficio importante, esta solución permite tener y manejar indicadores de gestión. Estos indicadores permiten conocer a detalle los procesos internos de la empresa y tener una idea de los puntos débiles y fuertes. Con esto se empieza un proceso de monitoreo de procesos críticos, análisis de los problemas generados y administración de recursos y procesos que permiten una mejor toma de decisión corporativa para mediante todo esto, optimizar el rendimiento. Según esta información se puede añadir que la aplicación de una solución de inteligencia de negocio nos brinda la capacidad de efectuar proyecciones y pronósticos de eventos que están por llegar, además de no olvidar la información histórica e importante que obtuvo la empresa. 
Con referencia del análisis de los tipos de información que son generados por una solución BI, (López, 2018) hace referencia a tres niveles. El primero es el nivel operacional el cual contiene datos transaccionales y están relacionados con el día a día operativo de la empresa. El segundo nivel es el táctico el cual es considerado el primer nivel del Bl y se encuentra vinculado a las jefaturas departamentales dentro de una empresa. El último nivel y el más alto es el estratégico el cual se relaciona directamente con las altas gerencias o direcciones de la empresa, para la toma de decisión.

Dentro de una solución de inteligencia de negocios, se puede categorizar en diferentes herramientas útiles para encaminar este proceso: Data management tools, Data discovery applications y las herramientas de Reporting. Según (Saguay, et al, 2017), en el mundo cada vez existe más demanda de datos, los mismos que siempre han existido en las empresas pero en estos últimos años, este aumento sucede debido al diverso acceso que existe con el internet y más aun con la invención de los dispositivos móviles que también generan información. Antes se realizaban el almacenamiento manual y físico de esta información, la cual hacía que su utilización, más que nada interpretación sea lenta y mínima. Con la tecnología, aún se archiva la información en carpetas, lo único que ahora son digitales, ya no son físicas, lo cual permite un acceso más rápido y oportuno (Erazo-Álvarez \& Narváez-Zurita, 2020).

El Data Warehouse según (Vaisman \& Zimányi, 2014) explica que es una base de datos particular que es usada para la toma de decisiones. Recoge información de bases de datos operacionales y otras fuentes de información y lo transforma en una nueva estructura que es usada en alcanzar objetivos establecidos. Esta información es confiable y constante ya que la información es recopilada cada que es generada. Además, tiene manera de archivar un historial de estos datos generados, lo cual es útil para poder analizar situaciones pasadas (Carreño-Godoy, et al., 2020).

Otra herramienta considerada dentro de los sistemas de Business Intelligence es el Data Mining. Para (Montaño-Moreno, et al., 2014), la data mining es el proceso de seleccionar de una gran cantidad de información, la más valiosa para una efectiva toma de decisión. Lo considera similar al proceso minero, donde de una gran cantidad 
de material crudo, se logra extraer lo más valioso como las piezas de oro puro. OLAP (On-Line Analytical Processing) o como se lo conoce en español como cubos de procesamiento analítico en línea, en este sentido, (Oña-Aldama \& Díaz-de-Armas, 2015), comentan que son herramientas dentro del sistema de inteligencia de negocios, el cual permite un análisis multidimensional y que permite a sus usuarios y empresas poder saber las causas de hechos ocurridos o de información compleja. Muchos utilizan esta herramienta por la rapidez de respuesta que este tiene.

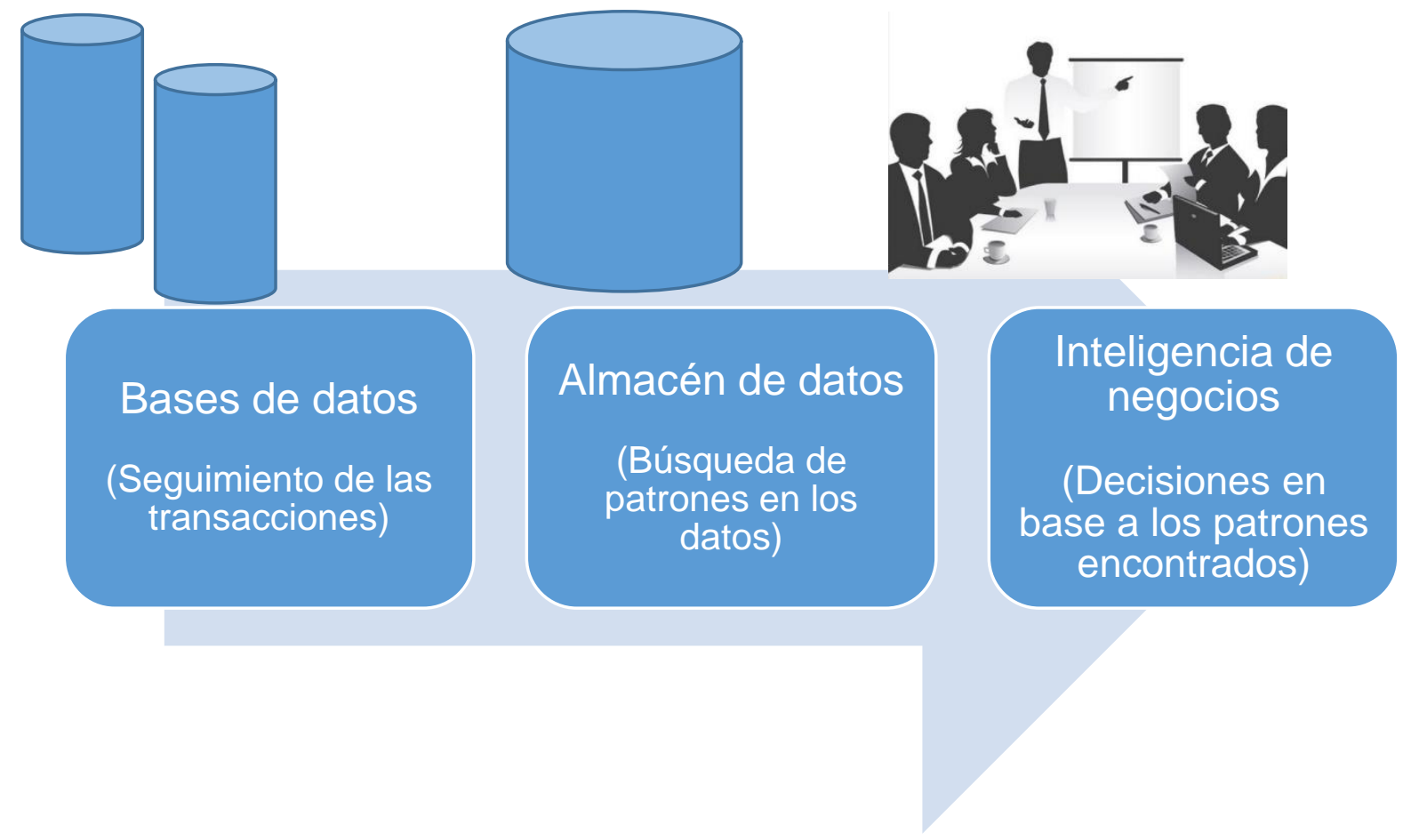

Figura 1. Proceso de una función de un almacén de datos.

Fuente: Cohen \& Asín (2009).

\section{El camino a seguir para implementar un sistema de business intelligence en una empresa}

La información digital hoy en día la encontramos en todo y con un crecimiento constante de los datos. La manera en la cual esta información o datos son utilizados, es la clave en el éxito de una empresa o sector ya que marca la diferencia del resto por la ventaja que uno adquiere al manejar esta información para el desarrollo de una 
estrategia. Además, ayuda a seleccionar la información que realmente es vital para la interpretación y generación de estrategias de manera rápida y continua (CorderoNaspud, et al., 2020).

Como importante referencia podemos incluir a (Chiavenato, 2006) donde expresa que información lo define como un conjunto de datos que contiene un significado o permite aumentar el conocimiento sobre un tema específico. Esta información es disponible para un uso inmediato y permite tomar mejores acciones y decisiones ya que aumenta el conocimiento de la misma. Para complementar esta definición (Toffler \& Toffler, 2006) exponen la diferenciación entre datos e información. Indican que los datos son elementos discretos y sin contexto, es cuando estos datos son puestos en contextos que se convierten en información.

Cuando los datos e información generada por el normal fluir de una empresa o sector son utilizados de manera adecuada, es cuando podemos tomar decisiones correctas y con bases sólidas, de esta manera generando una estrategia competitiva y valiosa. Apreciando que con adecuado manejo de información mediante los sistemas de Business Intelligence, esto ayuda al crecimiento y competitividad de cualquier empresa sin tomar en cuenta su tamaño (Montaño-Moreno, et al., 2014).

\section{METODOLOGÍA}

La investigación es de tipo descriptiva con diseño de campo no experimental, recopilándose información por medio de 690 funcionarios de la rama médica y odontológica, pública y privada, para poder entender sobre el manejo de información dentro del sistema de salud en estos dos sectores, siendo integrantes de la muestra poblacional, a través de encuesta y cuestionario de diversas opciones, siendo validado por juicio de expertos y coeficiente Alfa de Cronbach con un valor de 0,89 siendo confiable. Una vez recopilada la información, se procedió a procesar mediante estadística descriptiva con la finalidad de ser presentada en la sección de resultados. 


\section{RESULTADOS}

Aplicada la encuesta se determinan los siguientes resultados para dar a conocer un poco más la necesidad existente de una herramienta más adecuada y facilitadora para manejar la información de salud de los pacientes en el sector público y privado:

\section{Tabla 3}

Resultados de la encuesta realizada a los profesionales médicos y odontológicos del sector público y privado del ecuador

\begin{tabular}{|c|c|c|c|}
\hline \multirow{2}{*}{ Pregunta 1} & \multirow{2}{*}{ Opciones } & \multicolumn{2}{|c|}{ Resultados } \\
\hline & & Número & Porcentaje \\
\hline \multirow{4}{*}{$\begin{array}{l}\text { ¿Cómo define Ud. el } \\
\text { proceso de recopilación de } \\
\text { datos de salud de sus } \\
\text { pacientes? }\end{array}$} & Bueno & 332 & $48.12 \%$ \\
\hline & Regular & 208 & $30.14 \%$ \\
\hline & Malo & 150 & $21.74 \%$ \\
\hline & Totales & 690 & $100.00 \%$ \\
\hline \multirow{2}{*}{ Pregunta 2} & \multirow{2}{*}{ Opciones } & \multicolumn{2}{|c|}{ Resultados } \\
\hline & & Número & Porcentaje \\
\hline \multirow{4}{*}{$\begin{array}{l}\text { ¿Qué tanto de su tiempo } \\
\text { utiliza para ingresar de } \\
\text { manera manual la } \\
\text { información de salud de }\end{array}$} & Mucho & 497 & $72.03 \%$ \\
\hline & Poco & 179 & $25.94 \%$ \\
\hline & Nada & 14 & $2.03 \%$ \\
\hline & & & \\
\hline sus pacientes? & Totales & 690 & $100.00 \%$ \\
\hline \multirow{2}{*}{ Pregunta 3} & \multirow{2}{*}{ Opciones } & \multicolumn{2}{|c|}{ Resultados } \\
\hline & & Número & Porcentaje \\
\hline \multirow{3}{*}{$\begin{array}{l}\text { ¿Cree que el uso de } \\
\text { formularios de papel para } \\
\text { recopilar ésta información }\end{array}$} & Muy útil & 93 & $13.48 \%$ \\
\hline & Útil & 194 & $28.12 \%$ \\
\hline & No útil & 403 & $58.41 \%$ \\
\hline es útil? & Totales & 690 & $100.00 \%$ \\
\hline
\end{tabular}

\section{Pregunta 4}


Juan Antonio Palacios-Tapia; Juan Carlos Erazo-Álvarez; Cecilia Ivonne Narváez-Zurita; Mireya Magdalena Torres-Palacios

¿Qué tan importante es ésta información para una futura consulta del

Número

Muy importante

Poco importante

No importante

paciente?
611

58

21
Porcentaje

$88.55 \%$

$8.41 \%$

$3.04 \%$

Totales

690

$100.00 \%$

Totales

690

$100.00 \%$

Pregunta 5

Opciones

Muy necesario

¿Cree que el

Poco necesario

almacenamiento de ésta Innecesario

Resultados

Número

410

$59.42 \%$

129

$18.70 \%$

151

$21.88 \%$

información es necesario?

\section{Totales}

Pregunta 6

Opciones
Resultados

Número Porcentaje

$661 \quad 95.80 \%$

29

necesario para sustituir los

\begin{tabular}{cccc} 
formularios y lo físico? & Totales & 690 & $100.00 \%$ \\
\hline Pregunta 7 & Opciones & Número & Pesultados \\
¿Le gustaría que el & Si & 647 & $93.77 \%$ \\
historial de salud de sus & No & 43 & $6.23 \%$
\end{tabular}




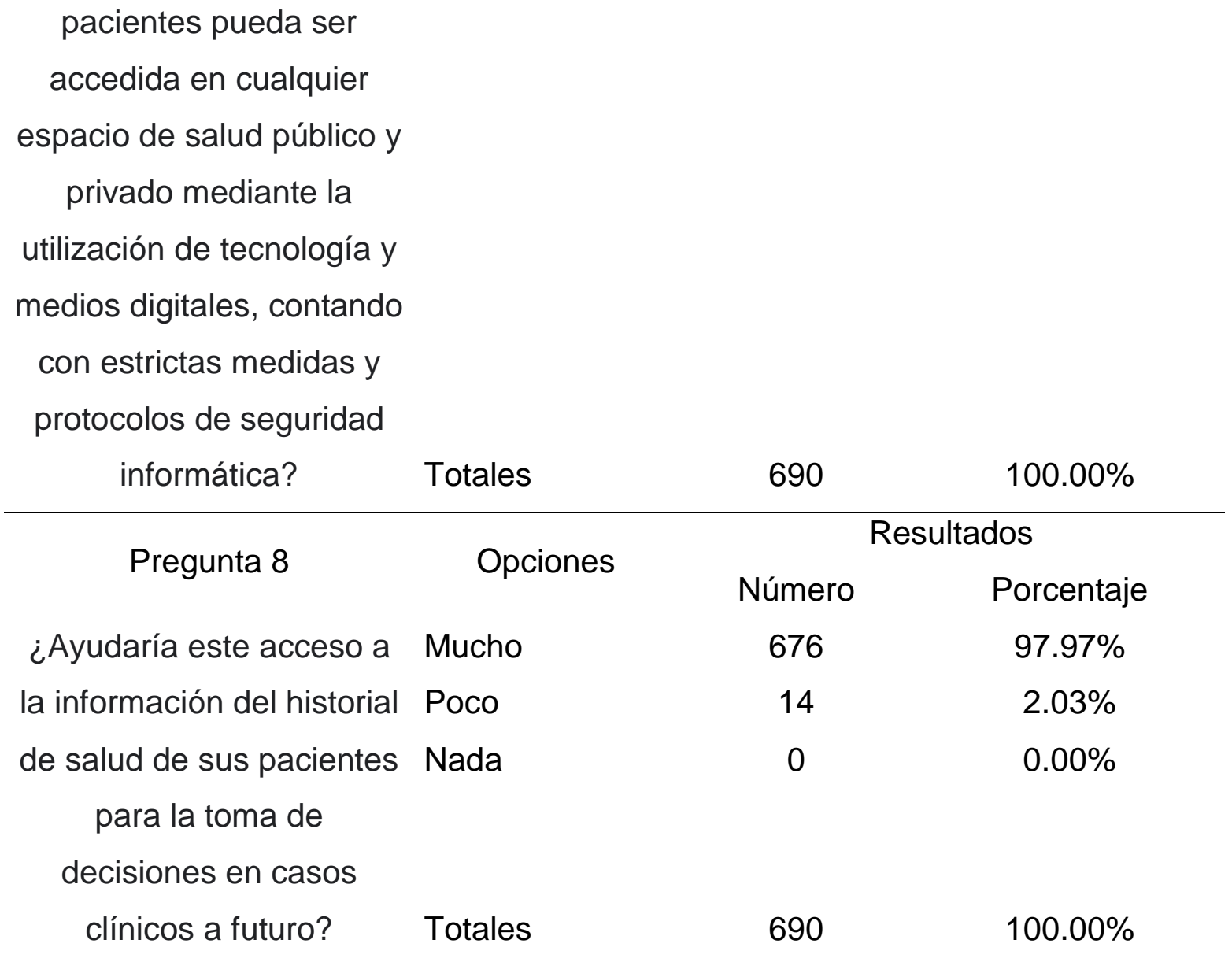

Fuente: Investigación de campo.

En relación a la primera pregunta, los profesionales de salud señalan que el proceso de recopilación de información de los pacientes tiene un nivel bueno y regular en su mayoría sin embargo al continuar con la siguiente pregunta se puede determinar que un $72.03 \%$ de los encuestados indican que este procedimiento toma mucho de su tiempo. Esto nos da a entender que ejecutar esto toma un gran porcentaje del tiempo que pudiera ser utilizado de manera más eficiente en la consulta o tratamiento que este por efectuarse al paciente.

Al continuar con la siguiente pregunta, los encuestados en un $58.41 \%$ contestó que el uso del papel que normalmente tienen como formularios y demás anexos, no son de gran utilidad. Analizando la pregunta cuatro, se determina que el $88.55 \%$ de los encuestados opinan que la información recopilada es de suma importancia para una consulta futura, con lo que podemos decir que el historial clínico de una persona es 
de vital importancia para decisiones o tratamientos médicos.

Cuando se analiza en la siguiente pregunta el valor que le ponen al almacenamiento de esta información, tenemos como resultado un $59.42 \%$ piensa que es muy necesario el archivo de los datos. Normalmente en los centros de salud públicos o privados esta información tiene un almacenamiento físico, por cuanto utilizan formularios de papel para la recopilación.

Con las últimas tres preguntas realizadas el objetivo es determinar una aceptación a la creación de una herramienta que les facilite el ingreso de la información, les permita continuo acceso y además pueda ser universal para los sectores públicos y privados. Obtuvimos un $95.80 \%$ de aceptación cuando se les propuso reemplazar los formularios físicos por la utilización de tecnología y medios digitales para el ingreso de datos. Además, con un $93.77 \%$ podemos ver que están de acuerdo con acceder a esta información de sus pacientes a nivel público y privado mediante tecnología que también proteja la misma con estándares de seguridad informática. Así mismo el $97.97 \%$ de los encuestados confirmaron que esta información ayudaría en la toma de decisiones en futuros casos clínicos por lo que consolidar en una herramienta universal para ambos sectores de la salud sería una decisión acertada. 
Juan Antonio Palacios-Tapia; Juan Carlos Erazo-Álvarez; Cecilia Ivonne Narváez-Zurita; Mireya Magdalena Torres-Palacios

\section{PROPUESTA}

La aplicación de una solución de inteligencia de negocios se ha convertido en una herramienta importante para el desarrollo de empresas o sectores que buscan consolidar datos, en tiempo real para una acertada toma de decisión, así contribuyendo con el crecimiento, la rentabilidad y generando una ventaja competitiva dentro del entorno en el que se encuentra. Para esto necesitas contar con los datos adecuados y confiables que viene de la información que día a día se suma y se puede utilizar como materia prima para desarrollar indicadores facilitando a una correcta y acertada toma de decisión.

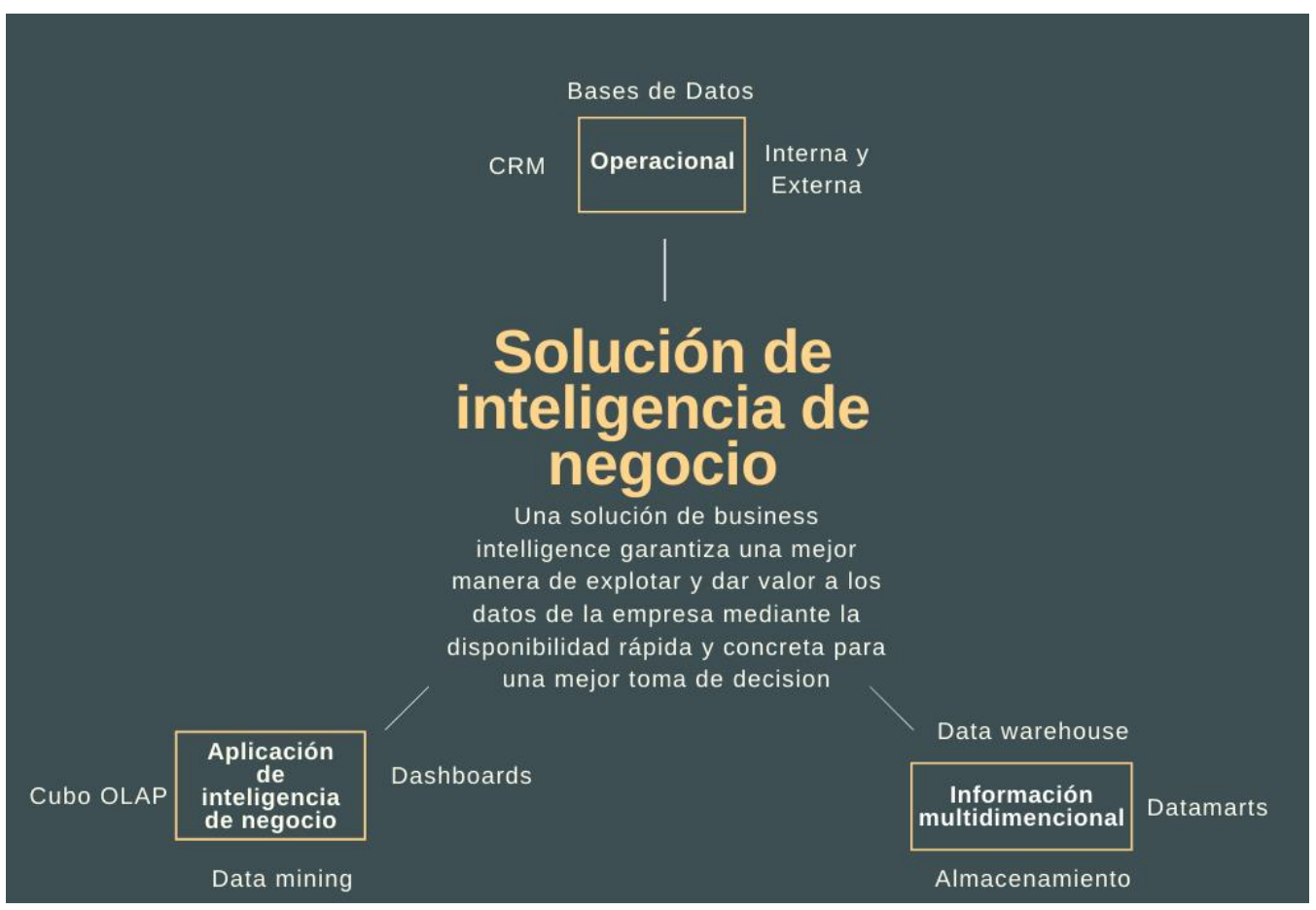

Figura 2. Esquema de propuesta inteligencia de negocio.

Fuente: Elaboración propia. 


\section{Operacional}

Al observar las necesidades confirmadas por la encuesta realizada a varios médicos y odontólogos dentro del ámbito de salud a nivel país, se genera esta propuesta de crear una herramienta de business intelligence que permita utilizar los datos de los pacientes que asisten a los diferentes centros de salud público y privado que de manera constante son ingresados de forma manual para guardarlos digitalmente. Aquí formando parte inicial del proceso de solución de inteligencia de negocios.

Cuando los pacientes ingresan en algunos de los hospitales o centros de salud pública o privada, o si éste asiste para una cita médica con algún especialista en su consultorio privado, desde el momento de su llegada, el personal de asistencia médica lleva a cabo la recopilación información básica del paciente, así como datos de su estado de salud. Aquí ingresan de forma escrita a formularios de papel, información que va desde el número de cédula, edad, sexo, peso, talla, tipo de sangre hasta los valores de presión arterial, frecuencia cardiaca, frecuencia respiratoria, saturación, temperatura, etc. Esta es una parte inicial de la información que se genera ya que cuando es momento de ser atendido, se valora la condición actual del paciente, así creando más referencias del mismo.

En esta primera etapa de la propuesta, lo más importante es llegar a tener ese contacto con el cliente o como es en este caso, el paciente, para reunir la información y convertirla en datos que utilizará el médico para dar solución a la condición médica que se le presente. La realidad que podemos observar es que la información en muchos de los casos es recopilada de manera escrita y que no es ingresada a algún sistema que la digitalice y sea de acceso fácil.

Al realizar este procedimiento, tenemos que complementarlo con un sistema de software que recoja esta información y la almacene mediante una segmentación por categorías que permitirá un ágil y rápido uso. Esto haría posible que se deje de usar los formularios de papel y la escritura manual de los datos generados en cada paciente. El nuevo formulario de registro que se utilizaría tiene que estar compuesto de la información más importante que vamos a necesitar y que complementen futuros procesos o decisiones clínicas o epidemiológicas. Es evidente que dicha información 
se debe manejar con total seguridad y confianza ya que esa tiene que ser consentida por el paciente y resguardada de usuarios ajenos a la práctica médica.

Dentro de la información que formará parte de esta base de datos existen los básicos, los de procedimientos médicos, altas hospitalarias, prescripción y entrega de medicamentos, etc. Siendo el ingreso digital de esta información de suma importancia para luego ser almacenada y procesada con el fin de ser complementada por una herramienta de inteligencia de negocio. En otras palabras, toda la información que de manera escrita se está siendo recopilada en muchos centros de salud, será convertida en información digital ingresada mediante el uso de una aplicación o software.

\begin{tabular}{|c|c|c|c|c|}
\hline \multicolumn{4}{|c|}{ Consulta fícha del paciente : Introduzca N Historia / Paciente / Cédula / Código..... } & $0102384^{* * *}$ \\
\hline \multirow{5}{*}{$\begin{array}{c}\text { Apellidos Paciente } \\
\text { Dirección } \\
\text { Ciudad } \\
\text { F. nacimiento } \\
\text { Sexo } \\
\end{array}$} & Ruiz Flores & & \multirow{5}{*}{$\begin{array}{c}\text { Nombre Paciente } \\
\text { Parroquia } \\
\text { Provincia } \\
\text { Codigo Paciente } \\
\text { Teléfono } \\
\end{array}$} & Karen \\
\hline & Ave. Don Bosco & & & Yanuncay \\
\hline & Cuenca & & & Azuay \\
\hline & $10 / 08 / 1985$ & 35 años-act. & & $6479-2$ \\
\hline & Mujer & & & 986758473 \\
\hline \multirow[t]{2}{*}{ F. consulta } & $15 / 05 / 2017$ & & \multirow[t]{2}{*}{ F. Alta } & $20 / 05 / 2017$ \\
\hline & Duración proceso & 5 dias & & \\
\hline Diagnóstico & \multicolumn{4}{|c|}{ DIARREA Y GASTROENTERITIS DE PRESUNTO ORIGEN INFECCIOSO } \\
\hline Tratamiento & \multicolumn{4}{|l|}{ Reposo y rehidratación. } \\
\hline Sintomas & \multicolumn{4}{|c|}{ Diarrea, calambres, náuseas, vómitos y febricula. } \\
\hline Medicamento Asignado & \multicolumn{4}{|c|}{ Ioperamida link, Amoxicilina } \\
\hline
\end{tabular}

Figura 3. Proceso operacional para ingreso de información. Fuente: Elaboración propia. 


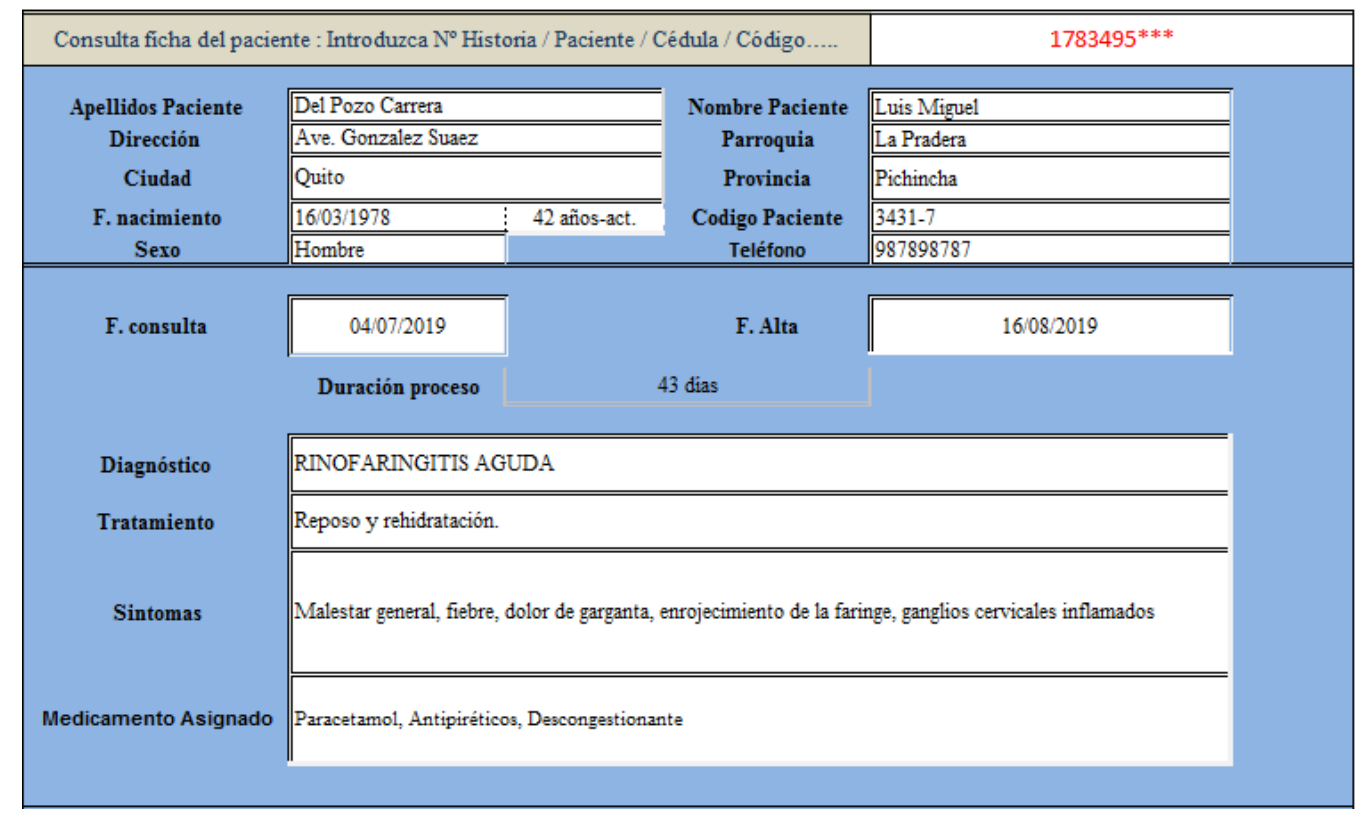

Figura 4. Proceso operacional para ingreso de información. Fuente: Elaboración propia.

\section{Información Multidimensional}

Una vez implementado el software que será útil para el ingreso continuo de todo tipo de datos que genera un paciente, estos serán enviados a reposar en un sistema de almacenamiento llamado data warehouse. Una data warehouse permite guardar de manera organizada la información que se genera diariamente con la finalidad de ser complementada para fines analíticos y de fácil y ágil acceso, además de integrarla dentro del proceso de toma de decisiones. En comparación con una base de datos común, una data warehouse permite extraer la información de la base de datos, la adecúa o transforma según la necesidad que se genere y lo archiva de tal forma que cuando se requiera, esta información estará disponible de manera inmediata.

Una data warehouse hoy en día puede ser alojada en el servidor de la entidad que la implemente o incluso como es de más frecuente uso hoy en día, mediante el espacio en una nube. Este sistema ocupa espacios digitales y no físicos como sucede en muchos centros de salud, donde tiene un cuarto solo para archivar miles y miles de hojas de expedientes o historias clínicas, siendo cada vez más obsoleto este tipo de 
almacenamiento y como anteriormente se mencionó no es almacenamiento de rápido y ágil acceso, mucho más cuando de importantes decisiones se trata como es la salud de una persona.

Una característica muy importante a destacar de la data warehouse es que no es volátil. Cuando información ingresa para ser almacenada, la misma no puede ser cambiada ni actualizada y mucho menos borrada. Convirtiendo esta característica en fundamental cuando hablamos de información sumamente importante como es el historial de salud de una persona. Aquí ingresan datos exactos de la condición médica y por ende esta información si bien puede variar con el tiempo, no genera cambio ni eliminación del histórico que se guarda, por lo que permite contar con esta información para desarrollar indicadores de salud de todo tipo.

La propuesta hasta este punto indica que, con el ingreso de un paciente, automáticamente, existen datos que son generados y serán de mucha utilidad para brindar la mejor atención y tratamiento médico u odontológico. Estos datos mediante la creación y uso de un software, serán ingresados para ser almacenados en una data warehouse. Previamente son sujetos al proceso de extracción, transformación y carga usando la herramienta ETL por sus siglas en inglés que significa extract, transform and load. Es donde aquí donde permitirá recopilar datos desde varias fuentes para ser luego consolidada en la data warehouse de esta manera obtener un mejor beneficio por medio del uso de información precisa y de alta calidad. 


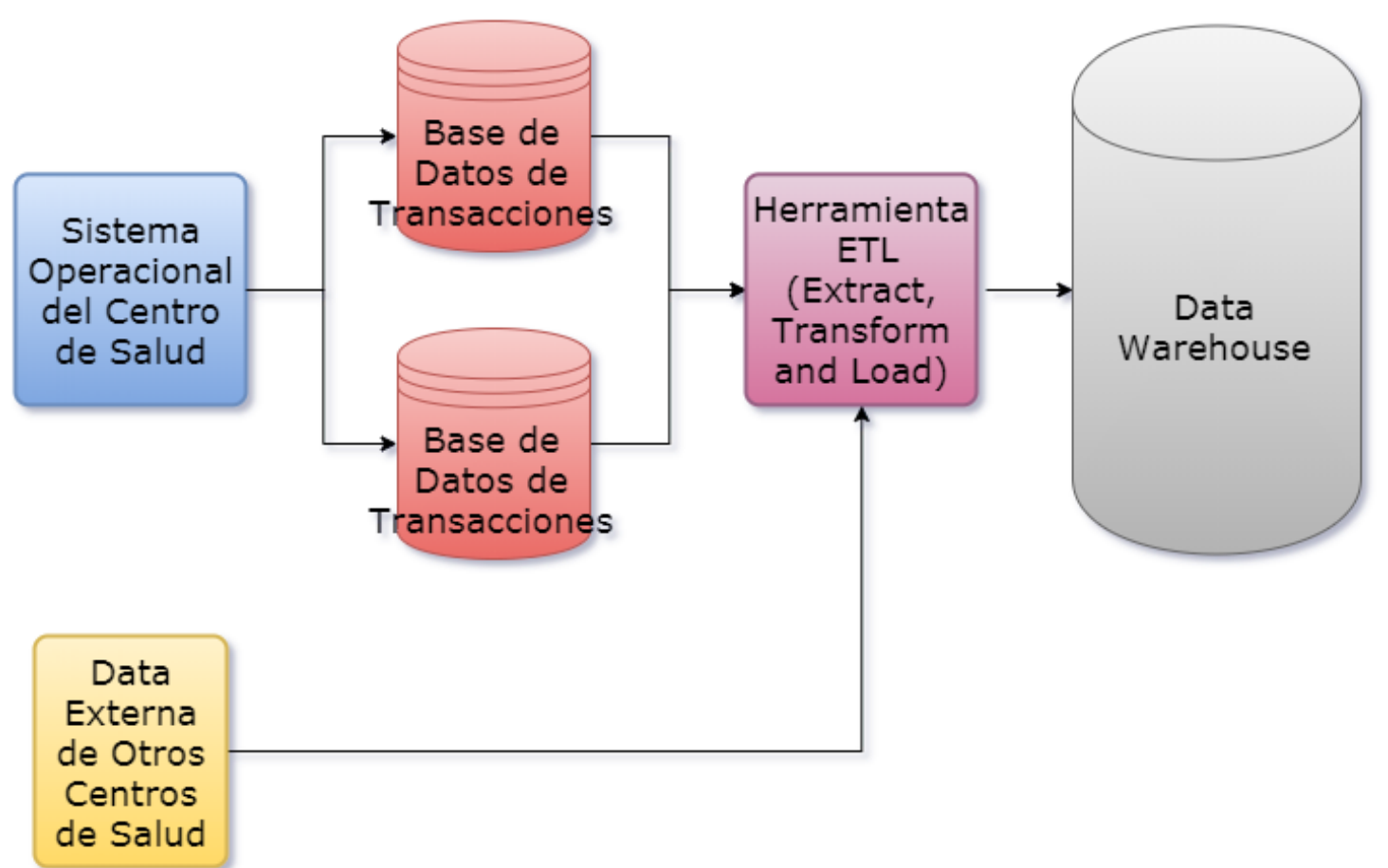

Figura 5. Flujo de información multidimensional con objetivo final, data warehouse. Fuente: Elaboración propia.

\section{Aplicación de Inteligencia de Negocios}

Los procesos de recolección, organización y almacenamiento de datos de los pacientes pudimos ver en las dos primeras etapas de nuestro esquema de inteligencia de negocio y es en esta última etapa que es esencial crear un complemento para una mejor toma de decisiones con bases confiables y precisas de los casos clínicos que se presenten. Los doctores de salud pública o privada, necesitan de información de sus pacientes para poder brindar atención, tratamiento, medicación o más aun cuando tienen que llevar a cabo algún tipo de procedimiento quirúrgico, es por este que vi la necesidad de usar la información como eje vital dentro de la salud.

El estructurar un modelo de almacenamiento como una data warehouse ayuda que los datos recopilados estén organizados y poder pasar a utilizar una herramienta de BI llamada dashboards o tableros de información. Es en esta herramienta que, tomando información histórica y organizada, el médico lo proyecta en su sistema usando indicadores que permitan analizar los antecedentes y la situación actual del 
paciente y de tal manera pueda tomar una decisión con bases más sólidas. La generación de dashboards ayuda a construir resultados o decisiones fundamentales. El uso de dashboards por parte del personal de salud permitirá ventajas como fácil acceso a la información desplegada en tableros personalizables, una comprensión sencilla de los datos para una mejor interpretación, además dejará analizar varios KPIs(Indicador clave de desempeño) de salud que mejoren el proceso de toma de decisión, y por último permite que el personal de salud tenga una mayor eficiencia en general para una mejor toma de decisión.

Otra herramienta que se puede implementar en un sistema BI para el sector de la salud es el On-line Analytical Processing o procesamiento analítico en línea. Esta herramienta permitiría procesar grandes cantidades de datos multidimensionales de manera ágil, siendo más útil cuando se quiera hacer una comparación histórica de estados de salud de pacientes con similares condiciones médicas o simplemente a cualquier pregunta en particular. Esto tendría más un propósito investigativocomparativo, siendo parte fundamental para una toma de decisión basado en un análisis de datos organizados y precisos. Las ventajas que se adquiere con la implementación de una herramienta $\mathrm{BI}$ al sector de salud son:

1. Ahorra tiempo en el ingreso de datos del paciente.

2. Crear un proceso estándar al momento de recopilar los datos ya que convierte en datos digitales y elimina la creación manual y en papel de los mismos.

3. Unifica la información más importante del paciente, siendo esa accesible en cualquier casa de salud que el paciente asista.

4. Permite crear y manejar indicadores importantes según la especialidad que esté tratando con el paciente para una precisa y ágil toma de decisión.

5. Con cada cita médica, el paciente reportara nuevos datos que serán ingresados al software y así manteniendo una base de datos actualizada.

6. Además de permitir una buena toma de decisión, esta herramienta brindará un complemento preventivo entorno a la salud de un paciente ya que se llegaría a analizar la evolución de salud, se pudiera detectar enfermedades a tiempo. 
Juan Antonio Palacios-Tapia; Juan Carlos Erazo-Álvarez; Cecilia Ivonne Narváez-Zurita; Mireya Magdalena Torres-Palacios

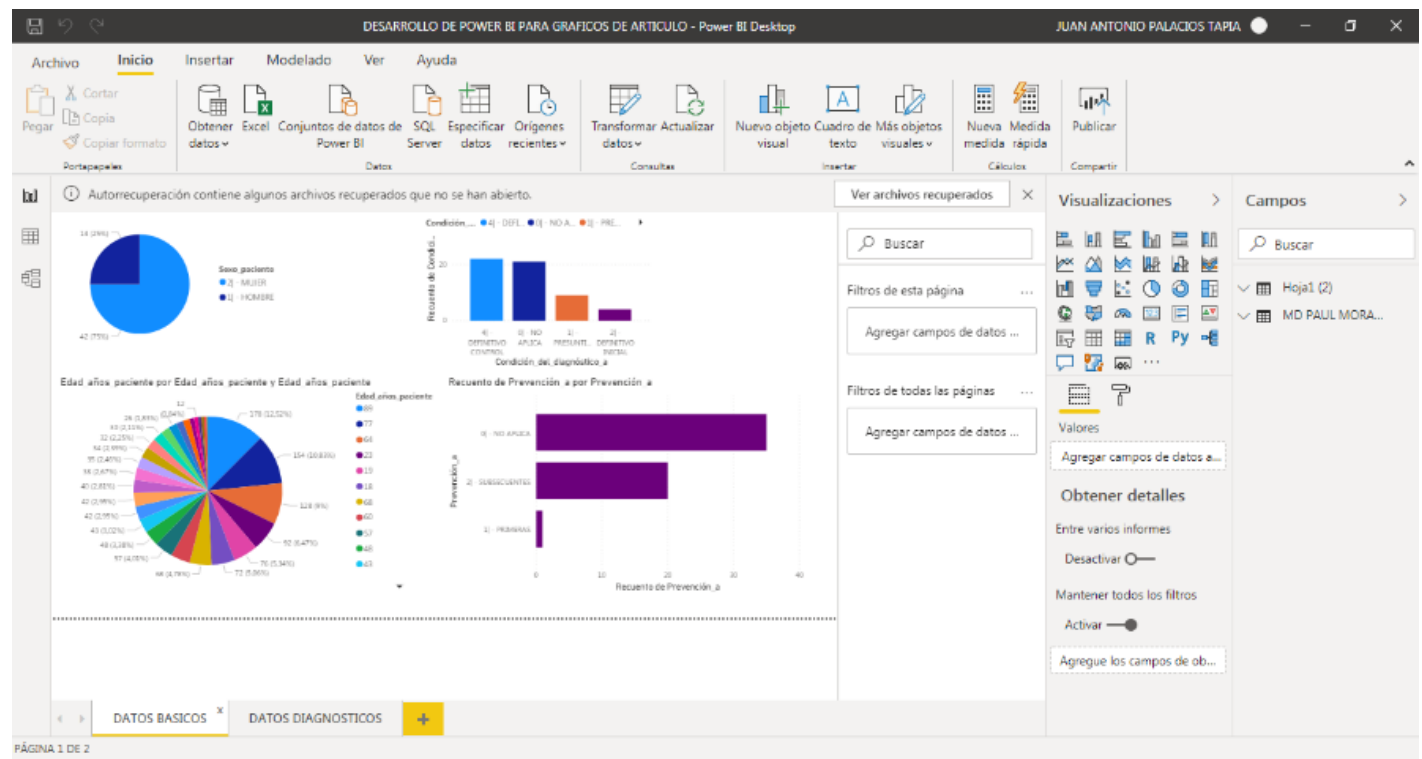

Figura 6. Consulta 1 de datos mediante herramienta Power BI. Fuente: Elaboración propia.

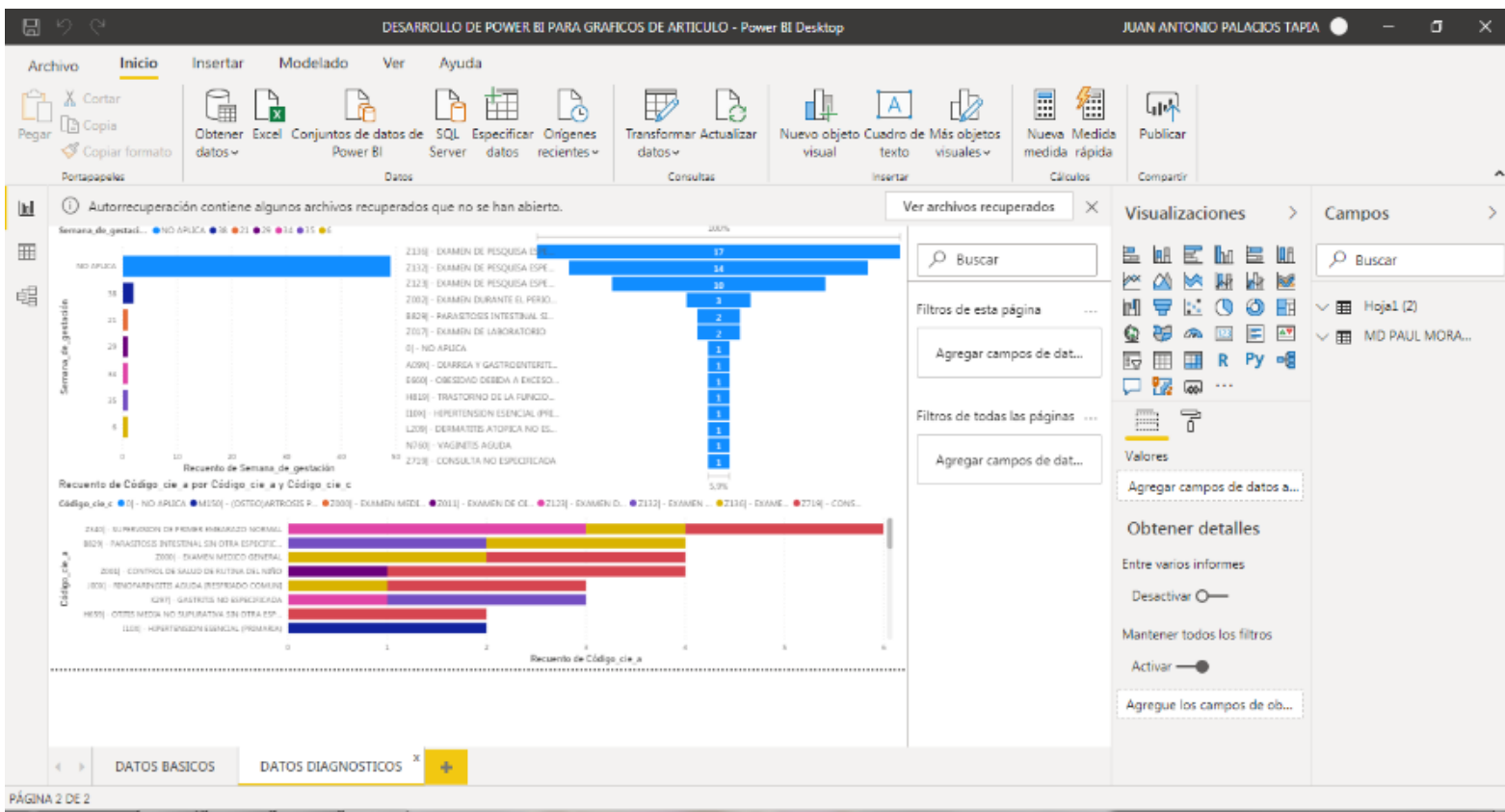

Figura 7. Consulta 2 de datos mediante herramienta Power BI. Fuente: Elaboración propia. 


\section{CONCLUSIONES}

El sector de la salud hoy en día es uno de los sectores que entró en una de las mayores fases de cambio generado por la pandemia que azota el mundo, que a su vez es analizada como un sector al cual se puede invertir, investigar e implementar muchas más soluciones que las que actualmente existen. Se habla de considerar a la salud como parte de un sector comunitario, zonal, nacional o incluso internacional. Hoy en día, al hablar del sector de la salud fácilmente podemos referirnos en un aspecto a nivel mundial. Esto sucede por la importancia que tiene la salud en nuestra sociedad y a nivel de empresas, si podemos catalogarlo así, por cuanto vemos la importancia de crear una solución de inteligencia de negocios, permitiendo que existan alternativas de cómo modernizar la parte administrativa-tecnológica-social, de esta manera generar procesos más rápidos, ágiles, que permitan también respuestas efectivas por parte de los expertos de salud. Asimismo, la implementación de este tipo de soluciones permite que en el sector público se puedan optimizar recursos por cuanto se estaría hablando de recorte en gasto de papelería, formularios, bodega, entre otros sectores.

Podemos comprar la idea de solución de BI expresada aquí, a un reporte del historial crediticio que manejan las instituciones financieras, ya que permite con autorización del usuario, revisar los créditos anteriores y actuales para de esta manera, la entidad financiera que consulta, pueda tomar la decisión de aprobar o denegar la solicitud de crédito y puesto en contexto para el sector de la salud, permitirá que el personal médico u odontológico, determine el mejor tratamiento o procedimiento de su paciente.

Se pretende utilizar un solo sistema a nivel público y privado para de esta manera consolidar los datos ingresados en varios sectores, hospitales, clínicas o especialidades, y así tener una herramienta precisa, confiable, eficiente, dinámica y ágil. El paciente puede confiar en que cuando acuda a cualquier casa de salud, ellos tengan su historial de salud y saber la manera de cómo tratar su condición actual. Como mencionamos anteriormente, también puede aportar para prevenir condiciones a futuro y así mantener a la persona más saludable. 
Esta herramienta como en todo proceso de implementación genera costos, sin embargo, se sugiere siempre considerar un costo beneficio de los resultados que se obtendría, al manejar una base de datos universal, tanto el paciente como el personal de salud, van a tener la confianza en los datos generados por la consulta realizada.

\section{FINANCIAMIENTO}

No monetario.

\section{AGRADECIMIENTO}

A los funcionarios de la rama médica y odontológica, pública y privada de la ciudad de Cuenca; por apoyar el desarrollo de la investigación.

\section{REFERENCIAS CONSULTADAS}

Ahumada-Tello, E., \& Perusquia-Velasco, J. (2016). Inteligencia de negocios: estrategia para el desarrollo de competitividad en empresas de base tecnológica. [Business intelligence: Strategy for competitiveness development in technology-based firms]. Contaduría y administración, 61(1), 127158. https://doi.org/10.1016/..cya.2015.09.006

Banco Interamericano de Desarrollo (BID, 2018). Mejor gasto para mejores vidas. Como America Latina y el Caribe puede hacer mas con menos. [Better spending for better lives. How Latin America and the Caribbean can do more with less]. Recuperado de https://n9.cl/mskxx.

Carreño-Godoy, M. J., Erazo-Álvarez, J. C., Narváez-Zurita, C. I., \& Moreno, V. P. (2020). La responsabilidad social en las empresas camaroneras [Social responsibility in shrimp companies]. Revista Arbitrada Interdisciplinaria Koinonía, 10(5), 455-482. http://dx.doi.org/10.35381/r.k.v5i10.702

Chiavenato, I. (2006). Introducción a la teoría general de la administración. [Introduction to the general theory of management]. Mexico, D.F.: McGraw Hill.

Cohen, D., \& Asín, E. (2009). Tecnologías de información en los negocios. [Information technologies in business]. México, D.F.: McGraw Hill. 
Cordero-Naspud, E. I., Erazo-Álvarez, J. C., Narváez-Zurita, C. I., \& Cordero-Guzmán, D. M. (2020). Soluciones corporativas de inteligencia de negocios en las pequeñas y medianas empresas [Corporate business intelligence solutions in small and medium enterprises]. Revista Arbitrada Interdisciplinaria Koinonía, 10(5), 483-513. http://dx.doi.org/10.35381/r.k.v5i10.703

Erazo-Álvarez, J. C., \& Narváez-Zurita, C. I. (2020). Medición y gestión del capital intelectual en la industria del cuero - calzado en Ecuador. [Measurement and management of intellectual capital in the leather industry - footwear in Ecuador]. Revista Arbitrada Interdisciplinaria Koinonía, 9(5), 437-467. http://dx.doi.org/10.35381/r.k.v5i9.662

Fuentes-Tapia, L., \& Valdivia-Pinto, R. (2010). Incorporación de elementos de inteligencia de negocios en el proceso de admisión y matrícula de una universidad chilena. [Incorporation of business intelligence elements in the admission and registration process of a chilean university]. Ingeniare. Revista chilena de ingeniería, 18(3), 383-394. https://dx.doi.org/10.4067/S0718$\underline{33052010000300012}$

Hernández, G. A. (2017). Arquitectura de software para la construcción de un sistema de cuadro de mando integral como herramienta de inteligencia de negocios. [Software Architecture for Building a Balanced Scorecard System as Business Intelligence Tool]. TIA, 5(2), pp. 143-152.

Howson, C. (2009). Business Intelligence: estrategias para una implementación exitosa. [Business Intelligence: strategies for a successful implementation]. México D.F.: McGraw-Hill.

Hugo-Cardenas, F. X., Jimenez-Rosero, C. E., Holovatyi, M., \& Lara-Pazos, P. E. (2020). El impacto de las redes sociales en la administración de las empresas. RECIMUNDO, 4(1),

173-182. https://doi.org/10.26820/recimundo/4.(1).enero.2020.173-182

Instituto Nacional de Estadística y Censos (2017). Registro Estadístico de Recursos y Actividades de Salud. [Statistical Register of Health Resources and Activities] Recuperado de https://n9.cl/zuog.

Léon, T. R. (2018). Ecuador: País Ineficiente En Salud Pública. [Ecuador: Inefficient Country In Public Health]. Recuperado de https://n9.cl/ehc7d

López, M. (2018). Trabajo Fin de Grado. Sistema para la toma de decisiones en la producción empresarial basado en Business Intelligence. [System for decision making in business production based on Business Intelligence]. Albacete, Albacete, España: Universidad de Castilla-La Mancha. Recuperado de https://n9.cl/jc12s7 
Juan Antonio Palacios-Tapia; Juan Carlos Erazo-Álvarez; Cecilia Ivonne Narváez-Zurita; Mireya Magdalena Torres-Palacios

Luhn, H. P. (1958). A Business Intelligence System. IBM JOURNAL. 314-319. Recuperado de https://n9.cl/ezwii

Martínez-Avella, M., \& Wills-Herrera, E. (2013). Creación de conocimiento en management: influencia de las características relaciónales y estructurales de las redes sociales. [Knowledge creation in management: the influence of the relational and structural characteristics of social networks]. Cuadernos de Administración, 26(46), 37-59.

Montaño-Moreno, J., Gervilla-García, E., Cajal-Blasco, B, \& Palmer, A. (2014). Data mining classification techniques: an application to tobacco consumption in teenagers. [Data mining classification techniques: an application to tobacco consumption in teenagers]. Anales de Psicología, 30(2), 633641. https://dx.doi.org/10.6018/analesps.30.2.160881

Mora, G. (2018). Siglo XXI economía de la información: gestión del conocimiento y Business Intelligence, el camino a seguir hacia la competitividad. [21st century information economy: knowledge management and Business Intelligence, the way forward towards competitiveness]. SIGNOS - Investigación En Sistemas De gestión, 10(2), 161-174. https://doi.org/10.15332/s2145-1389.2018.0002.09

Murillo-Junco, M, \& Cáceres-Castellanos, G. (2013). Business intelligence y la toma de decisiones financieras: una aproximación teórica. [Business intelligence and financial decision making: a theoretical approach]. Revista Logos, Ciencia \& Tecnología, 5(1),119-138.

Oña-Aldama, N, \& Díaz-de-Armas, M. (2015). Sistema de Inteligencia Empresarial. El arte de integrar la información aplicado a la Industria del Diagnóstico In Vitro. [Business intelligence system. The art of integrating information applied to the in vitro diagnosis industry]. Revista Cubana de Farmacia, 49(1), 4-17.

Rocha, G, \& Pereira-Mello, C. (2016). How to develop technology roadmaps? The case of a Hospital Automation Company. Production, 26(2), 345-358.

Rodríguez-Ponce, E, Pedraja-Rejas, L, \& Araneda-Guirriman, C. (2013). El proceso de toma de decisiones y la eficacia organizativa en empresas privadas del norte de Chile. [Decision-making process and organizational performance in private companies in northern Chile]. Ingeniare. Revista chilena de ingeniería, 21(3), 328-336.

Saguay, C, Proaño, R, Jácome, B, \& Aguirre, D. (2017). Implementación de una base de datos relacional difusa. Caso práctico: tutoría académica. [Implementation of a fuzzy relational database. Case study: academic tutoring]. Enfoque UTE, 8(Supl. 1), 77-91. https://dx.doi.org/10.29019/enfoqueute.v8n1.151 
Silva-Idrovo, R, Pino-Morán, F, \& Alejo-Machado, O. (2018). Influencia de la inteligencia de negocio en el marketing turístico. [Influence of business intelligence in tourism marketing]. Revista Universidad y Sociedad, 10(1), 326330.

Silva-Solano, L. E. (2017). Business Intelligence: un balance para su implementación. [Business Intelligence: a balance for its implementation]. InnovaG, (3), 27-36.

Toffler, A., \& Toffler, H. (2006). La Revolución de la Riqueza. [The Wealth Revolution]. Región y Sociedad, 21(40), 241-246.

Vaisman, A., \& Zimányi, E. (2014). Data Warehouse Systems Design and Implementation. Berlin: Springer. doi:10.1007/978-3-642-54655-6

Vanegas-Lago, E, \& Guerra-Cantero, L. (2013). Sistema de inteligencia de negocios para el apoyo al proceso de toma de decisiones. [Business intelligence system to support the decision making process]. Revista INGENIERÍA UC, 20(3), 2534.

\footnotetext{
(C2020 por los autores. Este artículo es de acceso abierto y distribuido según los términos y condiciones de la licencia Creative Commons Atribución-NoComercial-Compartirlgual 4.0 Internacional (CC BY-NC-SA 4.0) (https://creativecommons.org/licenses/by-nc-sa/4.0/
} 